\title{
Sleep, Emotional and Behavioural Difficulties in Children and Adolescents
}

\author{
Alice M. Gregory \\ Department of Psychology, Goldsmiths, University of London and Social, Genetic and \\ Developmental Psychiatry Centre, Institute of Psychiatry, London, U.K. T: +44 (0)20 \\ 7919 7959; F: +44 (0)20 7919 7873; E: a.gregory@gold.ac.uk \\ Avi Sadeh \\ The Adler Center for Research in Child Development and Psychopathology, Department \\ of Psychology, Tel Aviv University, Israel, T: +972 3-6409296;F: +972-3-6409547; E: \\ sadeh@post.tau.ac.il
}

Running head: Sleep, emotional and behavioural problems in youth For submission to: Sleep Medicine Reviews

Conflicts of interests: The authors declare no conflicts of interests.

Corresponding author: Alice Gregory 


\title{
Sleep, Emotional and Behavioural Difficulties in Children and Adolescents
}

\author{
Abstract \\ Links between sleep and psychopathology are complex and likely bidirectional. Sleep \\ problems and alteration of normal sleep patterns have been identified in major forms of \\ child psychopathology including anxiety, depression and attention disorders as well as \\ symptoms of difficulties in the full-range. This review summarizes some key findings \\ with regards to the links between sleep and associated difficulties in childhood and \\ adolescence. It then proposes a selection of possible mechanisms underlying some of \\ these associations. Suggestions for future research include the need to 1) use multi- \\ methods to assess sleep; 2) measure sleep in large-scale studies; 3) conduct controlled \\ experiments to further establish the effects of sleep variations on emotional and \\ behavioural difficulties; 4) take an interdisciplinary approach to further understand the \\ links between sleep and associated difficulties.

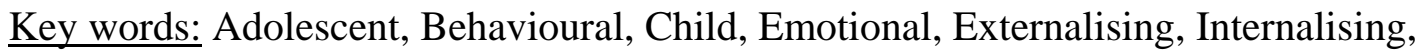 \\ Psychopathology
}


Abbreviations

\begin{tabular}{ll}
\hline Abbreviation & Full text \\
\hline ADHD & Attention Deficit Hyperactivity Disorder \\
ASD & Autism Spectrum Disorders \\
CBCL & Child Behavior Checklist \\
COMT & catechol-O-methyltransferase \\
DNA & Deoxyribonucleic Acid \\
EEG & Encephalogram \\
MAO-A & Monoamine Oxidize-A \\
MDD & Major Depressive Disorder \\
OCD & Obsessive Compulsive Disorder \\
PDD & Pervasive Developmental Disorders \\
PLMS & Periodic Limb Movements in Sleep \\
\hline
\end{tabular}




\section{Sleep, Emotional and Behavioural Disturbances in Children and Adolescents Introduction}

Sleep disturbances in children and adolescents are common. ${ }^{\text {e.g. 1;2 }}$ It is increasingly apparent that sleep disturbances are associated with both emotional (e.g. anxiety and depression) and behavioural (e.g. attention and conduct) difficulties in children and adolescents. ${ }^{3}$ Understanding more about co-occurring difficulties can facilitate understanding of the developmental progression of difficulties, aid researchers and clinicians in the early identification, prevention and treatment of difficulties as well as inform associated issues such as nosology (the classification of disorders). Given the known importance of sleep in youth, ${ }^{4}$ as well as the importance of having detailed knowledge of associations with other phenotypes, this review presents a selection of key empirical findings on the links between sleep and emotional and behavioural difficulties in children and adolescents. The review begins with a discussion of issues surrounding the definition and measurement of sleep disturbances. Concurrent links between sleep and emotional and behavioural difficulties are then described - followed by the presentation of longitudinal associations. A selection of possible mechanisms underlying associations are then described. The review ends with a description of future challenges for the field. These include the need to: 1) use multi-methods to assess sleep; 2) measure sleep in large-scale studies; 3) conduct controlled experiments to further establish the effects of sleep variations on emotional and behavioural difficulties; 4) take an interdisciplinary approach to further understand the links between sleep and associated difficulties. 


\section{Defining and measuring sleep disturbances}

Perhaps the biggest challenge when assimilating literature on sleep and associated difficulties is the lack of consensus regarding how to assess and define sleep disturbances. Three issues concerning the assessment of sleep disturbances are noteworthy. First, sleep disturbances can take many forms. For example, a distinction can be drawn between dyssomnias (such as insomnia) and parasomnias (such as sleep walking). Furthermore, the classification of such disorders vary, depending on the system being followed - and sleep disturbances are classified by the Diagnostic and Statistical Manual for Mental Disorders ${ }^{5}$ and the International Classification of Sleep Disorders. ${ }^{6}$ A second issue is that even within sleep difficulties (e.g. sleeplessness) there is lack of consensus concerning what constitutes a problem. ${ }^{7}$ A final issue concerns measurement. Sleep can be measured objectively using measures such as polysomnography (PSG) and actigraphy (for a discussion of the role of actigraphy in sleep medicine, see elsewhere), ${ }^{8}$ as well as subjectively using questionnaires and sleep diaries. There are strengths and weaknesses of each approach. For example, objective measures of sleep may fail to capture the subjective sense of having a problem with sleep; whereas the use of subjective measures alone does not allow investigation of sleep stage differences. Furthermore, different techniques can yield different results. For example, one study showed that youth with depression had sleep disturbances as defined using sleep diaries but not encephalogram (EEG) measures. ${ }^{9}$ Even within methodologies (e.g. questionnaires), conclusions can differ depending on the precise procedure used. Indeed, child self-reports of sleep disturbances have been shown to yield more sleep problems as compared to parental reports of their children's sleep ${ }^{2 ; 10}$ although interestingly when 
focusing on clinical samples the situation appears reversed (with parents reporting more difficulties than children). ${ }^{11 ; 12}$

Given a lack of consensus when defining and measuring sleep problems, studies to date have used numerous measures and definitions. Many studies have addressed a full range of 'sleep-related problems' rather than specific sleep disorders. This is particularly the case as a number of studies have capitalized on data already collected in large-scale (e.g. epidemiological) studies which have not assessed sleep thoroughly. This occurred partly because, up until recently, sleep appears to have been considered a secondary symptom of other problems rather than a phenotype worthy of consideration in its own right. In this review numerous conceptualizations of 'sleep disturbances', measured in different ways, are considered.

\section{Concurrent Associations}

\section{$\underline{\text { Sleep and Emotional Problems }}$}

Links between sleep disturbances, anxiety and depression in adulthood are well established. ${ }^{5}$ In contrast, only relatively recently has there been a wide interest in these associations within childhood and adolescence. Within this field, as with sleep disturbances, emotional problems have been conceptualized in different ways. While some studies combine symptoms of anxiety and depression into a single variable, ${ }^{3 ; 13}$ others distinguish between anxiety subtypes. ${ }^{14}$

Sleep and combined anxiety/ depression

Studies combining anxiety/ depression have reported associations with a range of different sleep disturbances. For example, in a non-clinical sample, trouble sleeping was associated with parent (but not teacher) reports of anxiety/depression in children when 
they were aged 6 years and again when they were aged 11 years. ${ }^{13}$ A further study reported that nightmares within childhood were associated with, amongst other symptoms, emotional difficulties. ${ }^{15}$ An additional study reported links between anxiety/depression and a composite measure of sleep disturbance in participants assessed between the ages of 4 and 15 years. $^{3}$

Studies examining associations between sleep and anxiety/ depression have focused on different age groups. Interestingly, there is some evidence for developmental changes in the magnitude of associations over time. For example, one study found that the association between sleep and anxiety/depression was greater in the children aged 11 $($ odds ratio $=9.7)$ as compared to when they were aged 6 years (odds ratio $=4.7){ }^{13}$ Interestingly, an increase in the magnitude of the association between sleep disturbance and anxiety/ depression from childhood (age 4 years, correlation $=.39$ ) to adolescence (age 13-15 years, correlation $=.52$ ) was reported in a further report. ${ }^{3}$ One possible explanation for this trend is that sleep disturbances may be more common in children than adolescents ${ }^{3}$ and hence perhaps more part of 'normal development' and less significant/ indicative of a problem (although it is important to note that sleep difficulties in childhood have been linked to various difficulties both concurrently and longitudinally). e.g. $3^{2}$

Sleep and anxiety

Further studies have focused on anxiety exclusively (rather than the combined anxiety/ depression phenotype). In one such community sample of adolescents, those with disturbing dreams had higher anxiety scores as compared to those who infrequently had such dreams. ${ }^{16}$ It is important to note that not all studies report robust associations 
between anxiety and sleep disturbances, and one community study of 8 year olds found that self-reported anxiety scores were higher in participants whose parents reported bedtime resistance than in those who did not, but not for the other seven aspects of sleep disturbances being studied (e.g. sleep onset delay). ${ }^{17}$

In addition to studies examining community samples, a number of reports have focused on clinical populations. One such study addressed the prevalence of sleep-related problems in youth with anxiety disorders - finding that one or more sleep-related problem was reported in $88 \%$ of these participants. ${ }^{18}$ While most studies do not differentiate between weekday and weekend sleep, this distinction was made in a sample comprising children with clinically-diagnosed anxiety and those who had never sought clinical intervention. ${ }^{19}$ Amongst interesting findings, anxious children reported going to bed later and having less sleep than non-anxious children on school nights. Furthermore, anxious children reported falling asleep more quickly and experiencing less night wakings during the night on weekends as compared to week nights.

Although most studies on the links between sleep and anxiety have focused on subjective measures of sleep (primarily using questionnaire measures), a few studies have considered objective measures. For example, a clinical study using objective measures of sleep in youth experiencing anxiety disorders ${ }^{20}$ found that when sleep was assessed using EEG, those with anxiety appeared to have poorer sleep than did those with depression and controls. For example, those with anxiety experienced more night wakings as compared to those with depression; and during the second night in the laboratory had a longer sleep latency as compared to both controls and those with depression. 
A number of studies have distinguished subtypes of anxiety and one study reported links between sleep and obsessive compulsive disorder. ${ }^{11}$ A further study examined EEG sleep profiles in adolescents diagnosed with obsessive compulsive disorder (OCD). ${ }^{21}$ There were differences in sleep between matched controls and those diagnosed with OCD (e.g. the latter slept for a shorter total period and less time was spent in stage 2 sleep). Despite this finding, it is noteworthy that in a community sample, the correlation between sleep and OCD symptoms in adolescents was not significant. ${ }^{14}$

Certain types of anxiety as compared to others may be more strongly associated with sleep. Indeed, in a community sample of 8 year olds, self-reported sleep disturbances appeared to be more strongly associated with certain types of anxiety (e.g. school phobia) than others (e.g. social phobias) ${ }^{22}$ A further study reported that while in childhood, sleep difficulties were associated with all types of anxiety examined; within adolescence, sleep disturbance appeared to be associated with certain types of anxiety (generalized anxiety, panic/ agoraphobia and social anxiety) but perhaps not others (obsessive compulsive symptoms and separation anxiety). ${ }^{14}$ Clinical studies comparing different anxiety subtypes also suggest that sleep difficulties may be more strongly associated with certain disorders (e.g. generalized anxiety disorder) as compared to others (e.g. social anxiety). ${ }^{18 ; 23}$

The issue of developmental change has been investigated with regards to the links between sleep and anxiety. One study found that in contrast to research focusing on combined anxiety/ depression, there did not appear to be a stronger association between sleep and anxiety in adolescents as compared to children. ${ }^{14}$ 


\section{Sleep and depression}

As with anxiety, studies focusing on 'pure' depression (rather than combined with anxiety) have found associations with subjective reports of sleep disturbances. Indeed, sleep complaints are common in prepubertal children and adolescents with depression (for a review, see elsewhere).$^{24}$ As when considering mixed anxiety/ depression, associations may be influenced by age. For example, in a comparison of children and adolescents with major depressive disorder (MDD) hypersomnia was reported less commonly in the children (16\%) as compared to the adolescents (34\%). ${ }^{25}$ Furthermore, in a study mentioned previously, in contrast to the association with anxiety, the association between symptoms of sleep problems and depression appeared to be weaker in children (correlation $=.26)$ as compared to adolescents $($ correlation $=.58) .{ }^{14}$

In addition to subjective reports of sleep disturbance, objectively assessed sleep disturbances have also been investigated in association with depression. Studies have revealed mixed results - with many studies failing to find objective sleep differences in children and adolescents with depression as compared to controls. ${ }^{9 ; 26 ; 27}$ Other studies have reported objective sleep differences between youth with and without depression. For example, in one study incorporating polysomnography (PSG), 21 children showing symptoms of depression were compared with 7 controls. ${ }^{28}$ The group with symptoms of depression experienced shorter rapid eye movement (REM) sleep latency, longer sleep latency and REM sleep duration and a higher number of night-wakings. This finding of shorter REM sleep latency (often considered a biological marker of endogenous depression) has been reported in other samples of children ${ }^{29 ; 30}$ and adolescents ${ }^{31}$ suffering depression. Overall, discrepancies between studies may be partly explained by 
differences in the age of participants and severity of depression symptoms (for a review, see elsewhere). ${ }^{32}$

Studies using other objective sleep assessment methods shed additional light on this topic. For example, an actigraphic study of rest-activity cycles in children with MDD found that in comparison to controls these children present damped circadian amplitude and lower light exposure and daytime activity levels. ${ }^{33}$ Such findings suggest that alternations in circadian rhythms could underlie changes in sleep architecture and overall clinical presentation in children and adolescents with depression.

Despite mixed reports, overall, it appears that associations between sleep and depression are stronger when focusing on subjective as compared to objective reports. Indeed, a study assessing both subjective and objective sleep in youth suffering MDD, found that depressed participants as compared to controls reported poorer sleep quality, claimed to experience a higher number of night awakenings, estimated that they were awake longer during the night and reported more difficulty waking. ${ }^{9}$ In contrast, sleep as assessed by EEG did not appear to be worse for depressed as compared to control participants. ${ }^{\text {see also, } 20}$ Similar findings have been reported for prepubertal children. ${ }^{\text {e.g. } 27}$

Considering all studies in this field, the only relatively consistent finding emerging from the cumulative subjective and objective sleep studies is related to increased sleep latency. ${ }^{24}$ Reduced REM sleep latency may also be found in depressed youth. $^{34}$ 


\section{$\underline{\text { Sleep and Behavioural Problems }}$}

Sleep and Attention Deficit Hyperactivity Disorder (ADHD).

The links between sleep and behavioural problems or disorders have also been under scrutiny over the last 3 decades. In this context, the topic of ADHD and related symptoms and behaviours has drawn most scientific attention. Because a number of review and meta-analytic papers have been published recently, ${ }^{\text {e.g. } 35 ; 36 ; 37}$ only a brief summary is included here.

Studies based on subjective reports have consistently reported that sleep problems are more common in children diagnosed with ADHD in comparison to controls. ${ }^{\text {e.g. } 37}$ These problems include bedtime resistance, sleep initiation difficulties, and nightwakings. ADHD and related symptoms have been consistently reported in children with sleep disorders including periodic limb movements in sleep (PLMS) and sleep-disturbed breathing (for a review, see elsewhere) ${ }^{38}$ PSG based studies comparing children with ADHD and controls have provided inconsistent results. With regards to sleep architecture, one meta-analytic review of PSG studies concluded that there were no consistent significant differences between children with ADHD and controls. ${ }^{36}$ The only consistent and significant finding in this meta-analysis was that children with ADHD are more likely than controls to suffer from PLMS. A second meta-analytic review concluded that children with ADHD have lower sleep efficiencies, more sleep stage shifts and increased apnea-hypopnea index in PSG studies. ${ }^{37}$ The discrepancy between the conclusions drawn is likely to be due in part to the different studies included in the two reports. Studies using actigraphy have also revealed some additional features including high variability in sleep schedules in children with ADHD as compared to controls. ${ }^{\text {e.g. }} 39$ 
Other actigraphic studies have shown that children with ADHD have shorter sleep time and that their sleep is more fragmented. ${ }^{\text {e.g. }}{ }^{40}$ Children with ADHD may also experience increased daytime sleepiness as compared to others. ${ }^{\text {e.g. }} 40$ Sleep deprivation or disruption may lead, in typically developing children, to compromised alertness and neurobehavioural functioning. ${ }^{\text {e.g. } 4 ; 41 ; 42}$ It is therefore possible that sleep problems contribute to the aetiology and exacerbation of symptoms of ADHD.

Sleep and aggression, conduct disorder and addiction.

Other behavioural problems such as aggression, $\underline{\text { conduct disorders, }}$ as well as addiction have received less attention, although there are indications that these disorders are also linked to sleep problems. Many studies, based on subjective or parental reports find associations between sleep problems, insufficient sleep and behavioural problems. For example, sleep-disordered breathing has been associated with behavioural problems including aggression. ${ }^{\text {e.g. } 43}$ Other sleep issues such as sleeping less than others have also been associated with behavioural difficulties..$^{\text {e.g. } 44 ; 45}$ Studies using objective measures of sleep (actigraphy) and teachers' or parental ratings of behaviour problems have also reported significant correlations between short sleep time or poor sleep quality and behavioural difficulties in school-age children. ${ }^{46 ; 47}$ Of note, a similar study in adolescents failed to find such relationships ${ }^{48}$ - again, potentially highlighting the importance of age when considering associations between sleep and associated difficulties. In adolescence,

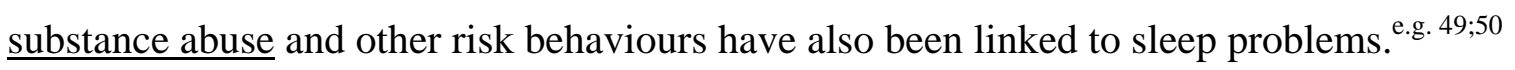

\section{$\underline{\text { Sleep and Other Difficulties }}$}

Pervasive developmental disorders (PDD), autism, or autism spectrum disorders (ASD) and related non-specified neurodevelopmental disorders have also been associated with 
significant sleep problems. ${ }^{\text {e.g. } 51 ; 52 ; 53}$ Studies of children with PDD or autism spectrum disorders consistently show higher prevalence of sleep problems in comparison to typically developing children or children with other developmental disabilities. Sleep problems are reported in between 25 and $80 \%$ of the children depending on sample composition and definition of sleep problems. ${ }^{53}$ Studies have also demonstrated associations between the severity of the autistic spectrum disorder and the severity of the sleep problems. ${ }^{54}$

Studies assessing children with ASD in comparison to controls using actigraphy have mostly reported poorer sleep as indicated by increased sleep latency and nightwakings, lower sleep efficiency and early morning risetime. ${ }^{\text {e.g. }}{ }^{55 ; 56}$ It is noteworthy that the identified unique sleep features of children with ASD are not always consistent across studies. Some studies report sleep schedule related problems (increased sleep latency, earlier morning risetime) whereas other studies report poor sleep quality (increased nightwaking, lower sleep efficiency).

PSG studies have also demonstrated unique sleep characteristics in children with ASD and related disorders. ${ }^{\text {e.g. }}$ 57;58 These reports have highlighted differences from typically developing children including reduced REM sleep latency and total sleep time. While findings are not all consistent across studies, the overall impression is that the sleep of children with ASD is substantially different from typically-developing children, and the unique characteristics of sleep in these children may be related to issues such as the severity of the neurodevelopmental disorder and comorbidity with other disorders and associated features. 
Sleep abnormalities have also been linked to a host of other difficulties. These include Tic disorder, where PSG studies suggest that sleep difficulties may include increased arousals during the sleep period. ${ }^{\text {e.g. } 59}$

\section{Longitudinal Associations}

In addition to highlighting concurrent associations, researchers have also investigated longitudinal associations between sleep and other phenotypes. Such studies encompass different time periods spanning a year or so ${ }^{60}$ to over a decade. ${ }^{61}$ These studies suggest that sleep disturbances in childhood or adolescence predict a whole host of later difficulties, ${ }^{\text {e.g. } 3 ; 50}$ with some studies reporting this link even when adjusting for the stability of difficulties over time. e.g. 3;62 $^{\text {3 }}$

$\underline{\text { Sleep and emotional problems }}$

Most longitudinal studies focusing on sleep have addressed the links with emotional problems. These studies suggest that sleep disturbances (conceptualized in various ways) in childhood or adolescence predict later anxiety ${ }^{63}$ and depression. ${ }^{62 ; 64}$ It is important to note that not all studies have reported significant associations. For example, in a further report it was found that sleep disturbances at 6 years of age were not associated with depression at 11 years of age, ${ }^{13}$ and in another, persistent sleep disturbances in childhood did not predict depression disorders in adulthood. ${ }^{63}$

Longitudinal studies of sleep and emotional difficulties have conceptualized sleep disturbances in various ways. A number of studies have defined sleep disturbance using items from the Child Behavior Checklist (CBCL) ${ }^{65}$, a measure of behavioural difficulties in youth which is commonly included in large-scale studies. ${ }^{\text {e.g. } 3 ; 45 ; 50}$ It is possible that certain items from the CBCL (e.g. 'sleeps less than others') are more robust predictors of 
later difficulties than others (e.g. 'sleeps more than others'). ${ }^{45}$ Few large-scale longitudinal studies have defined sleep thoroughly because sleep is typically not well assessed in such studies of health and development.

Most longitudinal studies of sleep and emotional difficulties have tested just one direction of effects (e.g. sleep predicting later difficulties). Such studies are limited in that they do not allow testing of the converse hypothesis (e.g. that early difficulties predict later sleep disturbances). Associations are likely to be bidirectional, although certain studies have found that sleep disturbances predict later emotional difficulties, but provide less support for the converse association. ${ }^{3 ; 66}$ Another study investigated the order of effects of insomnia, anxiety and depression in a community sample of adolescents. ${ }^{67}$ Retrospective reports indicated that anxiety disorders precede insomnia in $73 \%$ of comorbid cases whereas insomnia preceded depression in $69 \%$ of comorbid cases. The authors concluded that sleep difficulties may be associated with anxiety and depression in different ways (a conclusion supported by results from other studies). ${ }^{\text {e.g. } 63}$ One explanation for this general conclusion focuses on phenomenological differences between anxiety and depression. Indeed, hyperarousal, which according to the Tripartite model of anxiety and depression is associated with the former but not the latter difficulty, ${ }^{68}$ is sometimes considered an important feature of insomnia. ${ }^{69}$ Hyperarousal could therefore be a vulnerability factor for anxiety and insomnia (but not depression, although not all data support this suggestion). ${ }^{70}$

Sleep and behavioural problems

Fewer studies have examined longitudinal links between sleep and behavioural problems including attention problems ${ }^{\text {e.g. }} 71$ and addiction. ${ }^{49 ; 50}$ One study in this area showed that 
the persistence of sleep problems over a two-year period in school children predicts amongst other things, behaviour problems. ${ }^{72}$ A further study showed that mother reports of sleep problems experienced during childhood (3-8 years) predicted early onset of drug use (particularly in boys). ${ }^{50}$

Although previous longitudinal studies of sleep and associated phenotypes tend to ignore factors that could mediate and moderate associations - these issues need consideration. Indeed, one study highlighted sex differences in the association between sleep and later substance use, with sleep disturbances in childhood predicting alcohol, cigarette and marijuana use among adolescent boys but only alcohol use among adolescent girls. ${ }^{50}$ An additional study examining the longitudinal link between sleep and adjustment highlighted the importance of race and socioeconomic status as potential moderators of the association. ${ }^{73}$ Furthermore, a study of the trajectories of sleep disturbances and externalizing (aggression and rule-breaking) behaviours from ages 5 to 9 years, found that sleep and externalizing behaviour trajectories were only associated in children whose mothers had reported that they displayed high temperamental resistance to control during infancy. ${ }^{44}$

\section{Summary: sleep and associated difficulties}

A review of the literature reveals that the recent increase in interest in the links between sleep difficulties and associated problems in youth is warranted. Indeed, sleep disturbances have been associated with a whole host of other difficulties concurrently and may also represent risk indicators for the emergence of further problems later in life. Additional research is needed to confirm emerging trends (such as developmental changes in the magnitude of associations over time and the precise objective sleep 
differences between those with and without various disorders). An additional priority for future work is to understand the mechanisms underlying links between sleep and associated difficulties - and it is to this area of research that we now turn.

\section{Mechanisms underlying associations}

\section{Nosology.}

When explaining the associations between sleep disturbance and other difficulties it is important to reflect on the issue of nosology. Sleep disturbances are considered symptoms of a range of other disorders (including Generalized Anxiety Disorder and Major Depressive Disorder) as well as distinct diagnoses (see DSM, IV). ${ }^{5}$ This topic has been widely discussed and despite symptom overlap, there are strong arguments for not dismissing sleep difficulties as secondary to other difficulties. ${ }^{74}$ Indeed, sleep difficulties may precede other problems and treatment of sleep problems may reduce symptoms of other disorders. ${ }^{74}$ Regardless of whether sleep disturbances are considered symptoms of other disorders or separate diagnoses, it is important to try to understand this overlap in order to gain a fuller understanding of sleep problems themselves and the difficulties with which they are associated.

\section{$\underline{\text { Rater Bias. }}$}

One explanation for some of the associations reported concerns the methodological issue that sometimes the same raters (e.g. parents) report both sleep problems and associated traits. This allows for the possibility that rater bias (or response set) could account for some of the associations reported, although not all associations can be accounted for in this way. For example, some studies have reported associations between variables assessed by different raters (e.g. parent reports of sleep disturbances and child self-reports 
of emotional difficulties). ${ }^{17}$ Furthermore, other studies have examined links between objective measures of sleep and reports of psychiatric difficulties. ${ }^{20}$

\section{Twin Studies.}

One type of study which has been informative with regards to reasons underlying associated traits involves twins (for a discussion of twin studies, see elsewhere). ${ }^{75}$ Twin studies typically compare the similarity of identical and non-identical twins. This information can be used to draw inferences about the magnitude of genetic and environmental influences on the association between traits. There have been a handful of twin studies in children reporting on sleep and associated traits - and one study found that parent-reports of sleep disturbances in 3 year olds appeared to be genetically unrelated to all other scales assessed, including oppositionality; withdrawn/depressed behaviour; aggressive behaviour; anxious behaviour and overactivity. ${ }^{76}$ In contrast, common 'shared environmental factors' (i.e. those environmental factors which act to make individuals within a family alike) appeared to influence the whole range of difficulties. A further twin study of the links between sleep disturbance and depression symptoms in 8 year olds suggested that the association was largely explained by genes. ${ }^{17}$ When examining longitudinal associations between sleep at 8 and depression symptoms at 10 years, genes also appeared to play an important role. ${ }^{66}$ Twin studies focusing on adult participants have also examined the associations between sleep and associated traits e.g. $77 ; 78$ - although caution should be taken before extrapolating findings from adults to children. This is because of factors including developmental changes with regards to: sleep; and genetic influences on certain aspects of sleep. ${ }^{79}$ Although standard twin studies are informative in estimating the magnitude of genetic influences on traits, they do not 
tell us much about specific genes that influence traits, and this information typically comes from elsewhere (e.g. association and linkage studies).

Specifying genetic and environmental factors.

The specific genes implicated in the overlap between various phenotypes and sleep disturbance depends on the variable with which sleep is being associated. For example, genes involved in the serotonin pathways are likely to play a role in the associations between sleep and anxiety given the role that serotonin plays with regards to each phenotype. ${ }^{\text {e.g. } 80 ; 81}$ Monoamine oxidize A (MAO-A; an enzyme involved in the catabolism of monoamines) is a good candidate to further explore with regards to the links between sleep and aggression because it has been associated with both phenotype previously. ${ }^{82 ; 83}$ The functional polymorphism of the catechol-O-methyltransferase (COMT) gene has been implicated in the links between sleep and ADHD. ${ }^{84}$ Complex phenotypes are likely to be influenced by multiple genes of small effect size, hence there is a clear need to further specify genes involved in sleep disturbances and associations with other traits.

As with the need to further specify genes, it is also essential to elucidate additional environmental factors that account for the association between difficulties. Indeed, one study found that both family disorganization and maternal depression each correlated moderately with both sleep disturbance and anxiety symptoms in children aged 3 and 4 years and accounted for some of the association between the two difficulties. ${ }^{85}$ Other candidate environmental influences include being a bully victim, which is associated with both sleeping poorly and feeling sad ${ }^{86}$ and socioeconomic status which is associated with a whole host of difficulties including poor sleep. ${ }^{\text {e.g. 87;88 }}$ 
The role of parenting in sleep development and sleep problems have been repeatedly demonstrated, particularly in early childhood. ${ }^{\text {e.g. }}{ }^{89 ; 90}$ Within this arena, one study focusing on adolescents with a history of substance abuse found that perception of lower levels of parental involvement was associated with lower sleep efficiency and increased time in bed - an association that was mediated through psychological distress. ${ }^{91}$ A further study found that whereas in young children (aged 5-11 years), greater levels of parental warmth was associated with longer sleep during the week; in older participants (aged 12-19 years) stricter parental rules were associated with longer weekday sleep. ${ }^{92}$ Such findings emphasize the need to consider child sleep problems in the context of the family. ${ }^{93}$ As with sleep disturbances, different aspects of parenting are also known to be associated with emotional and behavioural difficulties. ${ }^{\text {e.g. }}{ }^{3}$ It is quite conceivable that parenting influences explain some of the shared variability between sleep and behaviour problems.

Stress and trauma have also been implicated in altered biobehavioural functioning and have also been associated with both psychopathology and sleep disorders. ${ }^{94 ; 95}$ Stressful life events or traumatic history could therefore be an additional bridge between sleep and psychopathology.

While genetic and environmental influences are commonly considered separately, it is likely the genetic and environmental factors do not work independently, but work in concert to exert their effects. Indeed, interactions between genes and environmental factors are shown for difficulties including sleep quality ${ }^{96}$ as well as various associated traits (including depression and behavioural difficulties). ${ }^{\text {see }} 97$

$\underline{\text { Pathways through which genetic and environmental factors work }}$ 
As well as further understanding genetic and environmental influences on sleep disturbances and associated variables, further research needs to elucidate the pathways by which these influences have their effects. Indeed, genetic/ environmental influences impact upon hormones as well as neural and psychological processes known to be associated with sleep and behavioural/ emotional problems.

\section{Hormones.}

Research has revealed melatonin abnormalities in children with $\mathrm{ADHD}^{98}$ or with ASD. ${ }^{\text {e.g. }}$ 99 These altered melatonin secretion patterns may explain sleep schedule difficulties seen in children with ASD and this hypothesis is supported by studies showing the positive effects of melatonin treatment on sleep in children with $\mathrm{ADHD}^{\text {e.g. } 100}$ and in children with ASD. ${ }^{\text {e.g. } 101}$

The environmental influence, light, influences melatonin production and blind individuals (with limited light perception) are more prone to suffer from sleep-schedule disorders than are fully sighted individuals. The dynamics of the links between sleepschedule disorder and aggressive behaviours were demonstrated in a case report of a blind adolescent. ${ }^{102}$

A further hormone likely to be involved in the association between sleep and difficulties in other areas is cortisol. Stress is a response to a perception of imminent threat which requires vigilance and sleep is an antagonistic behavior. Stress is associated with higher levels of corticotrophin releasing hormone, which may have insomnogenic actions (for a review, see elsewhere) ${ }^{103}$ - and the HPA axis which controls reactivity to stress is likely to be involved in the association between sleep and emotional difficulties. Neural processes. 
Neuroimaging studies on brain metabolism and activation following sleep deprivation and recovery sleep have shed light on the links between sleep and emotion regulation. ${ }^{104}$ A relevant neuroimaging study demonstrated that participants who had been sleep deprived for 35 hours showed a greater amygdala response to negative emotional stimuli as compared to participants who had not been sleep deprived. ${ }^{105}$ It was also found that sleep deprived participants, as compared to controls, showed weaker functional connectivity between the amygdala and the medial-prefrontal cortex (a brain region which is believed to help in regulating amygdala function) - suggesting that sleepdeprived participants had less ability than others to moderate emotional responses. The authors speculated that these results could support the proposal of causal links between sleep and mood disorders.

Psychological processes.

Pertinent information concerning psychological processes associated with sleep and cooccurring difficulties comes from studies of the effects of sleep deprivation on emotion recognition. ${ }^{\text {eg. } 106}$ van Der Helm and colleagues assessed the accuracy of facial emotional expression recognition in sleep deprived and control healthy adults. Their findings suggested that sleep deprivation resulted in reduction in the recognition of angry and happy emotional expressions (when these expressions were of moderate intensity). Taken together, studies focusing on sleep deprivation suggest that sleep is essential for proper emotional regulation and that sleep disruptions or insufficient sleep could lead to compromised emotional regulation which is one of the core features of many difficulties associated with sleep. ${ }^{104}$

\section{Summary and future challenges}


Sleep issues are ubiquitous in children with emotional and behavioural problems. These associations appear when measuring traits in the full range as well as at the extremes and occur when assessing associations both concurrently and longitudinally. Associations between sleep, emotional and behavioural difficulties are likely bidirectional, with sleep problems or insufficient sleep exacerbating emotional and behaviour difficulties; and, mood disturbances, anxiety and stress compromising sleep patterns.

Despite such general conclusions, the field is full of discrepancies. These appear to be associated with a host of factors including the age of participants - emphasizing the need to test hypotheses in different age groups. The precise definitions of sleep and emotional/ behavioural problems used also influence results. Indeed, where sleep has been assessed in large-scale epidemiological studies, suboptimal measures have often been used and there is a need to include more widely validated measures of sleep in studies of this type. More generally, refining definitions of psychopathology and behaviour problems has been an ongoing challenge in child psychiatry and related disciplines with significant implication for this area of research. Discrepancy in sleep assessment methods also explains contradictory findings - and videosomnography, actigraphy and PSG, which are the most common methods to assess sleep in the youth, can provide distinct information.

The mechanisms underlying associations are slowly being elucidated. Indeed, the use of different methodologies (e.g. prospective longitudinal studies; twin studies; experimental studies) has highlighted genetic and environmental factors on both emotional and behavioural difficulties and produced candidate hormonal, neural and psychological mechanisms through which genetic and environmental factors may exert 
their influences. Such studies have also highlighted the possibility that shared mechanisms (e.g. poor sleep and subsequent compromised emotional regulation) can lead to a range of difficulties. Studies in this domain also highlight the possibility that studying certain populations (e.g. those with visual impairment) may prove valuable in learning more about links between sleep and associated difficulties.

In order to further understand the links between sleep and emotional and behavioural difficulties, additional sleep intervention and manipulation studies are required - to examine the possibility of causal links and the therapeutic potential of sleep interventions in repairing emotional and behavioural outcomes. Interdisciplinary research (e.g. including twins in sleep manipulation studies and collecting Deoxyribonucleic acid (DNA) on those participating in such studies) will go some way towards providing a more comprehensive understanding of the links between sleep and associated traits. 


\begin{tabular}{|l|}
\hline Practice Points \\
\hline Research to date suggests that: \\
children and adolescents; \\
\hline 2. These associations are likely to be bidirectional and complex; \\
3. There are some inconsistencies in results depending on the age of the children and \\
whether sleep is assessed subjectively or objectively; \\
4. Both genetic and environmental factors are likely to contribute to the associations \\
5. Genetic and environmental factors are likely to have their influences via complex \\
pathways, influencing hormones as well as neural and psychological processes.
\end{tabular}




\begin{tabular}{|c|}
\hline Research Agenda \\
\hline To advance this important area of research, there is urgent need to: \\
\hline 1. Use multi-methods (i.e. objective and subjective measures) to assess sleep; \\
2. Measure sleep in large-scale longitudinal studies (e.g. epidemiological studies \\
\hline 3. Conduct controlled experiments to establish the effects of sleep variations on \\
emotional and behavioural difficulties; \\
4. Take an interdisciplinary approach to further understanding the links between \\
sleep and associated difficulties (e.g. examining environmental risk factors for \\
sleep disturbance in a genetically sensitive twin design).
\end{tabular}




\section{Acknowledgements}

Alice M. Gregory is supported by a Leverhulme Trust Fellowship. We thank Liat Tikotzky for her valuable comments. 


\section{$\underline{\text { References }}$}

(1) Kahn A, Van de Merckt C, Rebuffat E, Mozin MJ, Sottiaux M, Blum D, et al. Sleep problems in healthy preadolescents. Pediatrics 1989;84:542-6.

(2) Owens JA, Spirito A, McGuinn M, Nobile C. Sleep habits and sleep disturbance in elementary school-aged children. J Dev Behav Pediatr 2000;21:27-36.

(3) Gregory AM, O'Connor TG. Sleep problems in childhood: A longitudinal study of developmental change and association with behavioral problems. J Am Acad Child Adolesc Psychiatry 2002;41:964-71.

(4) Sadeh A, Gruber R, Raviv A. The effects of sleep restriction and extension on school-age children: What a difference an hour makes. Child Dev 2003;74:44455.

(5) American Psychiatric Association. Diagnostic and statistical manual of mental disorders. Washington, DC: American Psychiatric Association; 1994.

(6) American Academy of Sleep Medicine. International classification of sleep disorders: diagnostic and coding manual, 2nd edition. Westchester, IL: 2005.

(7) Wiggs L. Are children getting enough sleep? Implications for parents. Sociological Res Online 2007;12.

(8) Sadeh A, Acebo C. The role of actigraphy in sleep medicine. Sleep Med Rev 2002;6:113-24. 
(9) Bertocci MA, Dahl RE, Williamson DE, Iosif AM, Birmaher B, Axelson D, et al. Subjective sleep complaints in pediatric depression: A controlled study and comparison with EEG measures of sleep and waking. J Am Acad Child Adolesc Psychiatry 2005;44:1158-66.

(10) Gregory AM, Rijsdijk FV, Eley TC. A twin-study of sleep difficulties in schoolaged children. Child Dev 2006;77:1668-79.

(11) Storch EA, Murphy TK, Lack CW, Geffken GR, Jacob ML, Goodman WK. Sleep-related problems in pediatric obsessive-compulsive disorder. J Anx Dis 2008;22:877-85.

(12) Alfano CA, Pina AA, Zerr AA, Villalta IK. Pre-sleep arousal and sleep problems of anxiety-disordered youth. Child Psychiatry Hum Dev 2010;41:156-67.

(13) Johnson EO, Chilcoat HD, Breslau N. Trouble sleeping and anxiety/depression in childhood. Psychiatry Res 2000;94:93-102.

(14) Alfano CA, Zakem AH, Costa NM, Taylor LK, Weems CF. Sleep problems and their relation to cognitive factors, anxiety, and depressive symptoms in children and adolescents. Dep Anx 2009;26:503-12.

(15) Schredl M, Fricke-Oerkermann L, Mitschke A, Wiater A, Lehmkuhl G. Longitudinal study of nightmares in children: Stability and effect of emotional symptoms. Child Psychiatry Hum Dev 2009;40:439-49. 
(16) Nielsen TA, Laberge L, Paquet J, Tremblay RE, Vitaro F, Montplaisir J. Development of disturbing dreams during adolescence and their relation to anxiety symptoms. Sleep 2000;23:727-36.

(17) Gregory AM, Rijsdijk FV, Dahl RE, McGuffin P, Eley T. Associations between sleep problems, anxiety and depression in twins at 8 years of age. Pediatrics 2006;118:1124-32.

*(18) Alfano CA, Ginsburg GS, Kingery JN. Sleep-related problems among children and adolescents with anxiety disorders. J Am Acad Child Adolesc Psychiatry 2007;46:224-32.

(19) Hudson JL, Gradisar M, Gamble A, Schniering CA, Rebelo I. The sleep patterns and problems of clinically anxious children. Beh Res Therapy 2009;47:339-44.

*(20) Forbes EE, Bertocci MA, Gregory AM, Ryan ND, Axelson DA, Birmaher B, et al. Objective sleep in pediatric anxiety disorders and major depressive disorder. $\mathrm{J}$ Am Acad Child Adolesc Psychiatry 2008;47:148-55.

(21) Rapoport J, Elkins R, Langer DH, Sceery W, Buchsbaum MS, Gillin JC, et al. Childhood Obsessive-Compulsive Disorder. Am J Psychiatry 1981;138:1545-54.

(22) Gregory AM, Eley TC. Sleep problems, anxiety and cognitive style in schoolaged children. Infant Child Dev 2005;14:435-44.

(23) Alfano CA, Beidel DC, Turner SM, Lewin DS. Preliminary evidence for sleep complaints among children referred for anxiety. Sleep Med 2006;7:467-73. 
(24) Ivanenko A, Crabtree VM, Gozal D. Sleep and depression in children and adolescents. Sleep Med Rev 2005;9:115-29.

(25) Ryan ND, Puig-Antich J, Ambrosini P, Rabinovich H, Robinson D, Nelson B, et al. The clinical picture of major depression in children and adolescents. Arch Gen Psychiatry 1987;44:854-61.

(26) Dahl RE, Puig-Antich J, Ryan ND, Nelson B, Dachille S, Cunningham SL, et al. EEG sleep in adolescents with major depression - the role of suicidality and inpatient status. J Affect Disord 1990;19:63-75.

(27) Puig-Antich J, Goetz R, Hanlon C, Davies M, Thompson J, Chambers WJ, et al. Sleep architecture and REM-sleep measures in prepubertal children with major depression - A controlled study. Arch Gen Psychiatry 1982;39:932-9.

(28) Arana-Lechuga Y, Nunez-Ortiz R, Teran-Perez G, Castillo-Montoya C, JimenezAnguiano A, Gonzalez-Robles RO, et al. Sleep-EEG patterns of school children suffering from symptoms of depression compared to healthy controls. World J Biol Psychiatry 2008;9:115-20.

(29) Dahl RE, Ryan ND, Birmaher B, AlShabbout M, Williamson DE, Neidig M, et al. Electroencephalographic sleep measures in prepubertal depression. Psychiatry Res 1991;38:201-14.

(30) Emslie GJ, Rush AJ, Weinberg WA, Rintelmann JW, Roffwarg HP. Children with major depression show reduced rapid eye-movement latencies. Arch Gen Psychiatry 1990;47:119-24. 
(31) Lahmeyer HW, Poznanski EO, Bellur SN. EEG sleep in depressed adolescents. Am J Psychiatry 1983;140:1150-3.

(32) Alfano CA, Gamble AL. The role of sleep in childhood psychiatric disorders. Child Youth Care Forum 2009;38:327-40.

(33) Armitage R, Hoffmann R, Emslie G, Rintelman J, Moore J, Lewis K. Restactivity cycles in childhood and adolescent depression. J Am Acad Child Adolesc Psychiatry 2004;43:761-9.

(34) Lofthouse N, Gilchrist R, Splaingard M. Mood-related sleep problems in children and adolescents. Child Adolesc Psychiatric Clin N Am 2009;18:893-916.

(35) Owens JA. Sleep disorders and attention-deficit/hyperactivity disorder. Curr Psychiatry Rep 2008;10:439-44.

(36) Sadeh A, Pergamin L, Bar-Haim Y. Sleep in children with attention-deficit hyperactivity disorder: A meta-analysis of polysomnographic studies. Sleep Med Rev 2006;10:381-98.

(37) Cortese S, Faraone SV, Konofal E, Lecendreux M. Sleep in children with attention-deficit/hyperactivity disorder: Meta-analysis of subjective and objective studies. J Am Acad Child Adolesc Psychiatry 2009;48:894-908.

(38) Walters AS, Silvestri R, Zucconi M, Chandrashekariah R, Konofal E. Review of the possible relationship and hypothetical links between attention deficit hyperactivity disorder (ADHD) and the simple sleep related movement disorders, 
parasomnias, hypersomnias, and circadian rhythm disorders. J Clin Sleep Med 2008;4:591-600.

(39) Gruber R, Sadeh A. Sleep and neurobehavioral functioning in boys with attentiondeficit/hyperactivity disorder and no reported breathing problems. Sleep 2004;27:267-73.

*(40) Owens J, Sangal RB, Sutton VK, Bakken R, Allen AJ, Kelsey D. Subjective and objective measures of sleep in children with attention-deficit/hyperactivity disorder. Sleep Med 2009;10:446-56.

(41) Beebe DW, Gozal D. Obstructive sleep apnea and the prefrontal cortex: towards a comprehensive model linking nocturnal upper airway obstruction to daytime cognitive and behavioral deficits. J Sleep Res 2002;11:1-16.

(42) Gregory AM, Caspi A, Moffitt TE, Poulton R. Sleep problems in childhood predict neuropsychological functioning in adolescence. Pediatrics 2009;123:11716.

(43) Chervin RD, Dillon JE, Archbold KH, Ruzicka DL. Conduct problems and symptoms of sleep disorders in children. J Am Acad Child Adolesc Psychiatry 2003;42:201-8.

*(44) Goodnight JA, Bates JE, Staples AD, Pettit GS, Dodge KA. Temperamental resistance to control increases the association between sleep problems and externalizing behavior development. J Family Psychology 2007;21:39-48. 
(45) Gregory AM, Van den Ende J, Willis TA, Verhulst FC. Parent-reported sleep problems during development predicts self-reported anxiety/ depression, attention problems and aggression later in life. Arch Ped Adolesc Med 2008;162:330-5.

(46) Aronen ET, Paavonen EJ, Fjallberg M, Soininen M, Torronen J. Sleep and psychiatric symptoms in school-age children. J Am Acad Child Adolesc Psychiatry 2000;39:502-8.

(47) Sadeh A, Gruber R, Raviv A. Sleep, neurobehavioral functioning, and behavior problems in school-age children. Child Dev 2002;73:405-17.

(48) Moore M, Kirchner HL, Drotar D, Johnson N, Rosen C, ncoli-Israel S, et al. Relationships among sleepiness, sleep time, and psychological functioning in adolescents. J Pediatric Psych 2009;34:1175-83.

(49) Wong MM, Brower KJ, Fitzgerald HE, Zucker RA. Sleep problems in early childhood and early onset of alcohol and other drug use in adolescence. Alcoholism: Clin Exp Res 2004;28:578-87.

(50) Wong MM, Brower KJ, Zucker RA. Childhood sleep problems, early onset of substance use and behavioral problems in adolescence. Sleep Med 2009;10:78796.

(51) Doo S, Wing YK. Sleep problems of children with a pervasive developmental disorders: correlation with parental stress. Dev Med Child Neurol 2006;48:650-5. 
(52) Goldman SE, Surdyka K, Cuevas R, Adkins K, Wang L, Malow BA. Defining the sleep phenotype in children with autism. Dev Neuropsych 2009;34:560-73.

(53) Richdale AL, Schreck KA. Sleep problems in autism spectrum disorders: Prevalence, nature, \& possible biopsychosocial aetiologies. Sleep Med Rev 2009;13:403-11.

(54) Mayes SD, Calhoun SL. Variables related to sleep problems in children with autism. Res Autism Spectrum Dis 2009;3:931-41.

(55) Allik H, Larsson JO, Smedje H. Sleep patterns in school-age children with Asperger syndrome or high-functioning autism: A follow-up study. J Autism Dev Dis 2008;38:1625-33.

(56) Wiggs L, Stores G. Sleep patterns and sleep disorders in children with autistic spectrum disorders: insights using parent report and actigraphy. Dev Med Child Neurol 2004;46:372-80.

(57) Elia M, Ferri R, Musumeci SA, Del Gracco S, Bottitta M, Scuderi C, et al. Sleep in subjects with autistic disorder: a neurophysiological and psychological study. Brain Dev 2000;22:88-92.

*(58) Miano S, Bruni V, Elia M, Trovato A, Smerieri A, Verrillo E, et al. Sleep in children with autistic spectrum disorder: A questionnaire and polysomnographic study. Sleep Med 2007;9:64-70. 
(59) Kirov R, Kinkelbur J, Banaschewski T, Rothenberger A. Sleep patterns in children with attention-deficit/hyperactivity disorder, tic disorder, and comorbidity. J Child Psychol Psychiat 2007;48:561-70.

(60) Smedje H, Broman JE, Hetta J. Short-term prospective study of sleep disturbances in 5-8-year-old children. Acta Paediatrica 2001;90:1456-63.

(61) Ong SH, Wickramaratne P, Tang M, Weissman MM. Early childhood sleep and eating problems as predictors of adolescent and adult mood and anxiety disorders. J Affect Disord 2006;96:1-8.

(62) Roane BM, Taylor DJ. Adolescent insomnia as a risk factor for early adult depression and substance abuse. Sleep 2008;31:1351-6.

(63) Gregory AM, Caspi A, Eley TC, Moffitt TE, O'Connor TG, Poulton R. Prospective longitudinal associations between persistent sleep problems in childhood and anxiety and depression disorders in adulthood. J Abnorm Child 2005;33:157-63.

(64) Roberts RE, Roberts CR, Chen IG. Impact of insomnia on future functioning of adolescents. J Psychosom Res 2002;53:561-9.

(65) Achenbach TM, Edelbrock C. Manual for the Child Behaviour Checklist and revised child behaviour profile. Vermont: Burlington; 1983. 
(66) Gregory AM, Rijsdijk FV, Lau JF, Dahl RE, Eley TC. The direction of longitudinal associations between sleep problems and depression symptoms: a study of twins aged 8 and 10 years. Sleep 2009;32:189-99.

*(67) Johnson EO, Roth T, Breslau N. The association of insomnia with anxiety disorders and depression: Exploration of the direction of risk. J Psychiatr Res 2006;40:700-8.

(68) Clark LA, Watson D. Tripartite model of anxiety and depression: Psychometric evidence and taxonomic implications. J Abnorm Psychol 1991;100:316-36.

(69) Bonnet MH, Arand DL. Hyperarousal and insomnia. Sleep Med Rev 1997;1:97108.

(70) Gauthier AK, Chevrette T, Bouvier H, Godbout R. Evening vs. morning wake EEG activity in adolescents with anxiety disorders. J Anx Dis 2009;23:112-7.

*(71) O'Callaghan FV, Al Mamun A, O'Callaghan M, Clavarino A, Williams GM, Bor W, et al. The link between sleep problems in infancy and early childhood and attention problems at 5 and 14 years: Evidence from a birth cohort study. Early Hum Dev 2010;86:419-24.

(72) Quach J, Hiscock H, Canterford L, Wake M. Outcomes of child sleep problems over the school-transition period: Australian population longitudinal study. Pediatrics 2009;123:1287-92. 
*(73) El-Sheikh M, Kelly RJ, Buckhalt JA, Hinnant JB. Children's sleep and adjustment over time: The role of socioeconomic context. Child Dev 2010;81:870-83.

(74) Harvey AG. Insomnia: Symptom or diagnosis? Clin Psychol Rev 2001;21:103759.

(75) Plomin R, DeFries JC, McClearn GE, McGuffin P. Behavioral genetics. Fifth ed. New York: Worth Publishers; 2008.

(76) Van den Oord EJCG, Boomsma DI, Verhulst FC. A study of genetic and environmental effects on the co-occurrence of problem behaviors in three-yearold twins. J Abnorm Psychol 2000;109:360-72.

(77) Heath AC, Eaves LJ, Kirk KM, Martin NG. Effects of lifestyle, personality, symptoms of anxiety and depression, and genetic predisposition on subjective sleep disturbance and sleep pattern. Twin Res 1998;1:176-88.

(78) Kendler KS, Heath AC, Martin NG, Eaves LJ. Symptoms of anxiety and depression: Same genes, different environments? Arch Gen Psychiatry $1987 ; 44: 451-7$.

(79) Jones KHS, Ellis J, von Schantz M, Skene DJ, Dijk DJ, Archer SN. Age-related change in the association between a polymorphism in the PER3 gene and preferred timing of sleep and waking activities. J Sleep Res 2007;16:12-6.

(80) Jouvet M. Biogenic amines and states of sleep. Science 1969;163:32-41. 
(81) Lesch KP, Bengel D, Heils A, Zhang Sabol S, Greenburg BD, Petri S, et al. Association of anxiety-related traits with a polymorphism in the serotonin transporter gene regulatory region. Science 1996;274:1527-31.

(82) Alia-Klein N, Goldstein RZ, Kriplani A, Logan J, Tomasi D, Williams B, et al. Brain monoamine oxidase a activity predicts trait aggression. J Neuroscience 2008;28:5099-104.

(83) Brummett BH, Krystal AD, Siegler IC, Kuhn C, Surwit RS, Zuchner S, et al. Associations of a regulatory polymorphism of monoamine oxidase-A gene promoter (MAOA-uVNTR) with symptoms of depression and sleep quality. Psychosom Med 2007;69:396-401.

(84) Gruber R, Grizenko N, Schwartz G, Ben Amor L, Gauthier J, de Guzman R, et al. Sleep and COMT polymorphism in ADHD children: Preliminary actigraphic data. J Am Acad Child Adolesc Psychiatry 2006;45:982-9.

(85) Gregory AM, Eley TC, O'Connor TG, Rijsdijk FV, Plomin R. Family influences on the association between sleep problems and anxiety in a large sample of preschool aged twins. Person Ind Diff 2005;39:1337-48.

(86) Williams K, Chambers M, Logan S, Robinson D. Association of common health symptoms with bullying in primary school children. B M J 1996;313:17-9.

(87) Buckhalt JA, El-Sheikh M, Keller P. Children's sleep and cognitive functioning: Race and socioeconomic status as moderators of effects. Child Dev 2007;78:21331. 
(88) Miech RA, Caspi A, Moffitt TE, Wright BRE, Silva PA. Low socioeconomic status and mental disorders: A longitudinal study of selection and causation during young adulthood. Am J Sociology 1999;104:1096-131.

(89) Bell BG, Belsky J. Parents, parenting, and children's sleep problems: Exploring reciprocal effects. Br J Dev Psych 2008;26:579-93.

(90) Sadeh A, Tikotzky L, Scher A. Parenting and infant sleep. Sleep Med Rev 2010;14:89-96.

(91) Cousins JC, Bootzin RR, Stevens SJ, Ruiz BS, Haynes PL. Parental involvement, psychological distress, and sleep: A preliminary examination in sleep-disturbed adolescents with a history of substance abuse. J Family Psych 2007;21:104-13.

(92) Adam EK, Snell EK, Pendry P. Sleep timing and quantity in ecological and family context: A nationally representative time-diary study. J Family Psych 2007;21:4-19.

(93) Dahl RE, El-Sheikh M. Considering sleep in a family context: Introduction to the special issue. J Family Psych 2007;21:1-3.

(94) Charuvastra A, Cloitre M. Safe enough to sleep: Sleep disruptions associated with trauma, posttraumatic stress, and anxiety in children and adolescents. Child Adolesc Psychiatric Clin N Am 2009;18:877-891.

(95) Sadeh A. Stress, trauma, and sleep in children. Child Adolesc Psychiatric Clin of N Am 1996;5:685-700. 
(96) Brummett BH, Krystal AD, shley-Koch A, Kuhn CM, Zuchner S, Siegler IC, et al. Sleep quality varies as a function of 5-HTTLPR genotype and stress. Psychosom Med 2007;69:621-4.

(97) Moffitt TE, Caspi A, Rutter M. Strategy for investigating interactions between measured genes and measured environments. Arch Gen Psychiatry 2005;62:47381.

(98) van der Heijden KB, Smits MG, van Someren EJW, Gunning WB. Idiopathic chronic sleep onset insomnia in attention-deficit/hyperactivity disorder: A circadian rhythm sleep disorder. Chronobiology International 2005;22:559-70.

(99) Melke J, Botros HG, Chaste P, Betancur C, Nygren G, Anckarsater H, et al. Abnormal melatonin synthesis in autism spectrum disorders. Mol Psychiatry 2008;13:90-8.

*(100) van der Heijden KB, Smits MG, van Someren EJW, Ridderinkhof KR, Gunning WB. Effect of melatonin on sleep, behavior, and cognition in ADHD and chronic sleep-onset insomnia. J Am Acad Child Adolesc Psychiatry 2007;46:233-41.

(101) Paavonen EJ, Nieminen-von Wendt T, Vanhala R, Aronen ET, von Wendt L. Effectiveness of melatonin in the treatment of sleep disturbances in children with Asperger disorder. J Child Adolesc Psychopharm 2003;13:83-95.

(102) Sadeh A, Klitzke M, Anders TF, Acebo C. Sleep and aggressive-behavior in a blind, retarded adolescent - A concomitant schedule disorder - case-study. J Am Acad Child Adolesc Psychiatry 1995;34:820-4. 
(103) Richardson GS. Human physiological models of insomnia. Sleep Med 2007;8:S9S14.

*(104) Walker MP, van der Helm E. Overnight therapy? The role of sleep in emotional brain processing. Psychol Bull 2009;135:731-48.

(105) Yoo SS, Gujar N, Hu P, Jolesz FA, Walker MP. The human emotional brain without sleep - a prefrontal amygdala disconnect. Curr Bio 2007;17:R877-R878.

(106) van der Helm E, Gujar N, Walker MP. Sleep deprivation impairs the accurate recognition of human emotions. Sleep 2010;33:335-42. 